\title{
Composite $Y$ internal thoracic artery-saphenous vein grafts: Short-term angiographic results and vasoreactive profile
}

\author{
Mario Gaudino, MD \\ Francesco Alessandrini, MD \\ Claudio Pragliola, MD ${ }^{\mathrm{a}}$ \\ Nicola Luciani, MDa \\ Carlo Trani, MD ${ }^{\mathrm{b}}$ \\ Francesco Burzotta, MD \\ Fabiana Girola, MD ${ }^{\mathrm{a}}$ \\ Giuseppe Nasso, MD \\ Giovanni Guarini, MDa \\ Gianfederico Possati, MD ${ }^{a}$
}

Background: The angiographic patency of composite $\mathrm{Y}$ internal thoracic arterysaphenous vein grafts has not been investigated in detail.

Methods: Twenty-five patients who received composite $\mathrm{Y}$ internal thoracic arterysaphenous vein grafts had control angiography and vasoactive challenges with serotonin, acetylcholine, and isosorbide dinitrate at a mean of $2.5 \pm 1.2$ years after surgery.

Results: The perfect patency rate of composite $\mathrm{Y}$ internal thoracic artery-saphenous vein grafts was $72 \%(18 / 25)$. The distal portion of the internal thoracic artery was stringed in 4 patients and occluded in 2 . The saphenous branch of the composite $Y$ internal thoracic artery-saphenous vein grafts was found patent in all patients except 1. No failures were reported in the proximal tract of the internal thoracic artery. The distal tract of the internal thoracic artery showed reduced capacity of endotheliummediated relaxation.

Conclusion: The short-term patency of composite $\mathrm{Y}$ internal thoracic artery-saphenous vein grafts is suboptimal and markedly influenced by distal runoff and native flow competition.

From the Departments of Cardiac Surgery ${ }^{\mathrm{a}}$ and Cardiology, ${ }^{\mathrm{b}}$ Catholic University, Rome, Italy.

Received for publication May 13, 2003; revisions received June 27, 2003; accepted for publication July 7, 2003.

Address for reprints: Mario Gaudino, MD, Divisione di Cardiochirurgia, Policlinico Universitario A. Gemelli, Largo A. Gemelli 8, 00168 Rome, Italy (E-mail: mgaudino @ tiscali.it).

J Thorac Cardiovasc Surg 2004;127:1139-44 $0022-5223 / \$ 30.00$

Copyright $\odot 2004$ by The American Association for Thoracic Surgery

doi:10.1016/j.jtcvs.2003.07.051

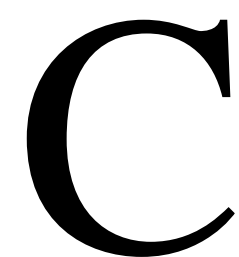
omposite $\mathrm{Y}$ internal thoracic artery-saphenous vein grafts (YITASVs) represent an unusual type of coronary artery bypass conduit. As YITA-SV combines the best and the worst conduit used in coronary surgery, obvious theoretical perplexities exist regarding its use in uncomplicated cases. The only suggested scenario for the adoption of this type of graft configuration is the necessity to minimize ascending aorta manipulation in patients with multivessel coronary disease and diffusely atherosclerotic ascending aorta. ${ }^{1-4}$ The very selected and relatively uncommon indication and the fact that YITA-SVs have often been used as last resort in extreme cases explain the absence of systematic angiographic studies of these conduits and the lack of information on their patency in the years after surgery.

During the past 48 months we adopted this type of graft configuration in 28 patients. As YITA-SV can represent an interesting model of internal thoracic artery flow diversion and "hybrid" vascular architecture, and due to the limited information available on this issue, we decided to ask all our patients to undergo control 


\section{TABLE 1. Preoperative characteristics of the patients}

\begin{tabular}{lr}
\hline Male/female & \multicolumn{1}{c}{$19 / 6$} \\
Mean age & $75.5 \pm 8.9$ \\
History & \\
$\quad$ hypertension & $15(60 \%)$ \\
$\quad$ diabetes & $19(76 \%)$ \\
$\quad$ smoking & $7(28 \%)$ \\
$\quad$ dyslipemia & $12(48 \%)$ \\
$\quad$ family history of CAD & $5(20 \%)$ \\
Peripheral vasculopathy & $21(84 \%)$ \\
Chronic obstructive pulmonary disease & $18(72 \%)$ \\
Previous stroke & $13(52 \%)$ \\
Previous AMI & $21(84 \%)$ \\
Canadian Cardiovascular Society angina class & \\
I & $10(40 \%)$ \\
II & $7(28 \%)$ \\
III & $8(32 \%)$ \\
Number of diseased vessels & \\
2 & $8(32 \%)$ \\
3 & $12(48 \%)$ \\
LMCA & $5(20 \%)$ \\
Left ventricular ejection fraction & $0.42 \pm 0.11$ \\
\hline
\end{tabular}

$A M I$, Acute myocardial infarction; $C A D$, coronary artery disease; $L M C A$, left main coronary artery.

angiography and to try to elucidate the vasoreactive characteristics of YITA-SV by means of endovascular vasoactive challenges.

\section{Patients and Methods \\ Patient Population}

From January 1999 to December 2002 YITA-SVs were used in 28 consecutive patients. These patients represent $0.7 \%$ of all coronary artery bypass $(\mathrm{CABG})$ procedures performed during that period. The decision to adopt this unusual type of conduit was based on the necessity to avoid aortic manipulation (due to severe ascending aorta atherosclerosis, too short ascending aorta, or presence of an ascending aorta prosthesis) coupled with the impossibility of using the additional arterial conduits in use at our institution: the right internal thoracic and the radial artery. All patients survived surgery and were discharged from the hospital in good condition. Twentyfive of these patients agreed to undergo control angiography and represent the objects of this study. The main preoperative features of the patients and the contraindications to use additional arterial conduits are reported in Tables 1 and 2. Patients were in their eighth decade, had poor left ventricular function and limited functional capacity, and had a high incidence of associated pathologies.

\section{Surgical Technique}

The operation performed was isolated CABG in 19 patients, CABG and aortic valve replacement in 4 , and CABG plus aortic valve and ascending aorta replacement in the remaining 2. All isolated CABG procedures were performed on the beating heart. The YITA-SV was performed using the standard technique. After completion of the proximal anastomosis the internal thoracic artery (ITA) was always anastomosed to the left anterior descending artery (LAD) and the saphenous vein (SV) to either a diagonal branch (1 patient), an obtuse marginal artery (11 patients), a
TABLE 2. Contraindications to the use of additional arterial conduits

\begin{tabular}{lr}
\hline Contraindications to BITA use & 16 \\
Insulin-dependent diabetes & 14 \\
Severe chronic obstructive pulmonary disease & 1 \\
Previous mediastinal irradiation & 9 \\
Obesity & \\
Contraindications to RA use & 19 \\
Lack of preoperative evaluation of forearm vasculature & 6 \\
Insufficient ulnar compensation &
\end{tabular}

$B I T A$, Bilateral internal thoracic arteries; $R A$, radial artery.

posterolateral branch (8 patients), or the posterior descending artery (5 patients).

In all patients 2 coronary anastomoses were performed; myocardial revascularization was complete in 13 patients $(52 \%)$ and incomplete in the remaining 12. In consideration of the poor systemic and cardiac status of these patients and of the considerable operative risk (see Table 1), this high degree of incomplete revascularization was judged acceptable.

\section{Angiographic Protocol}

Patients were studied in a fasting state, after premedication with oral diazepam $(10 \mathrm{mg})$. Selective YITA-SV angiography was performed by percutaneous left radial $(\mathrm{n}=24)$ or right femoral approach $(\mathrm{n}=1)$. Thoracic artery catheters $(5 \mathrm{~F}$ or $6 \mathrm{~F}$; Boston Scientific, Natick, Mass) were used as appropriate to obtain optimal YITA-SV visualization and selective contrast medium injection. Multiple angiographic views were obtained to detect significant stenosis at any YITA-SV level; Thrombosis in Myocardial Infarction flow grade was visually estimated separately by 2 different observers.

Pharmacological stimulation was then started according to a previously described protocol, ${ }^{5}$ using serotonin, isosorbide dinitrate, acetylcholine, and isosorbide dinitrate, as follows.

Serotonin hydrochloride $10^{-5} \mathrm{~mol} / \mathrm{L}$ (ICN Pharmaceuticals Inc, Costa Mesa, Calif) was selectively injected into the YITA-SV graft at a rate of $3 \mathrm{~mL} / \mathrm{min}$ for 3 minutes; at the end of the serotonin challenge $2 \mathrm{mg}$ of isosorbide dinitrate was injected into the conduit. After a 20-minute period acetylcholine chloride $10^{-6}$ $\mathrm{mol} / \mathrm{L}$ (Miovisin, Farmigea, Italy) was selectively injected into the YITA-SV at a rate of $1.5 \mathrm{~mL} / \mathrm{min}$ for 3 minutes; again at the end of the acetylcholine infusion $2 \mathrm{mg}$ of isosorbide dinitrate was injected into the graft. Drug infusion was always performed under electrocardiogram and invasive blood pressure monitoring. At the end of each step of the protocol a cine run was performed, keeping fixed angiographic view.

Digital angiograms were then analyzed using computerized quantitative angiography (Medis, Neuen, The Netherlands). Due to the complex vascular architecture of the graft and for a more detailed angiographic analysis of the conduit, the YITA-SV was divided in 3 vascular segments: proximal ITA (proximal to the saphenous vein anastomosis), distal ITA (distal to the saphenous vein anastomosis), and SV graft. The 3 segments were measured in end-diastolic frames after proper catheter calibration as follows:

- proximal ITA: 10 to $15 \mathrm{~mm}$ proximal to the SV anastomosis; 


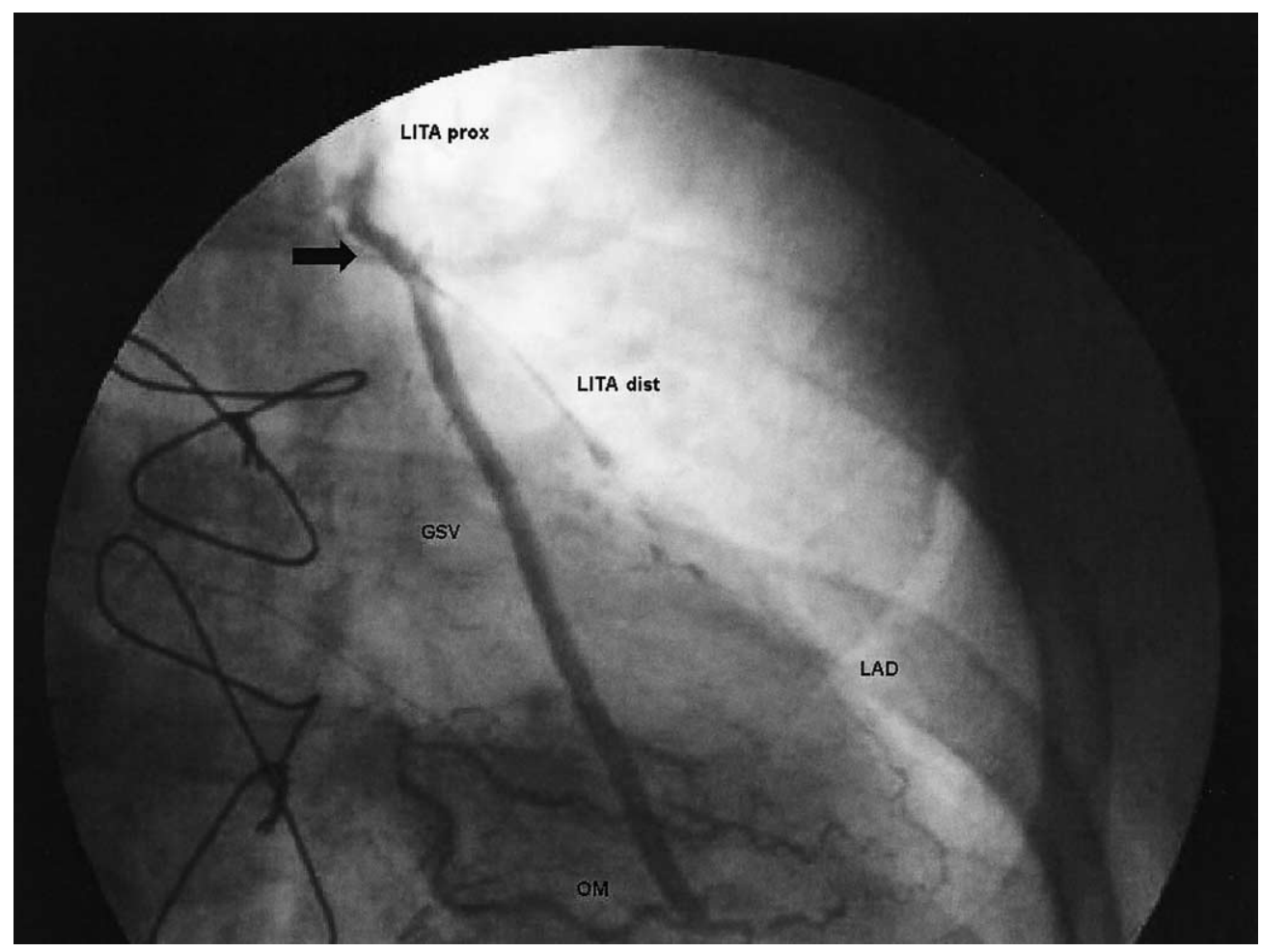

Figure 1. Stringed distal internal thoracic artery in a $Y$ internal thoracic artery-saphenous vein graft. The internal thoracic artery shows diffuse narrowing of its distal portion. Arrow indicates thoracic artery-saphenous vein anastomosis. GSV, Great saphenous vein; LAD, left anterior descending artery; LITA prox, proximal left internal thoracic artery; LITA dist, distal left internal thoracic artery.

- distal ITA: 10 to $15 \mathrm{~mm}$ distal to the SV anastomosis;

- SV graft: 10 to $20 \mathrm{~mm}$ distal to anastomosis with the ITA. Perfect patency was defined as the absence of any irregularity at the level of all 3 vascular segments examined and of all 3 anastomoses.

Patients did not received vasoactive medications in the 24 hours before the procedure. Written informed consent was obtained from each patient.

\section{Statistical Analysis}

Chi-square or Fisher exact tests were used to compare discrete parameters. Analysis of Variance (ANOVA) for repeated measures was used to test differences after vasoactive challenges; post hoc comparison was performed by Neuman-Keuls test. Analysis was conducted using the software Statistica for Windows 4.1 (Statsoft, Inc, Tulsa, Okla).

\section{Results}

\section{Angiographic Patency}

Control angiography was performed at a mean of $2.5 \pm 1.2$ years after surgery. Eighteen of the 25 grafts were perfectly patent $(72 \%)$; the distal portion of the ITA was stringed in 4 patients (Figure 1) and occluded in 2 (Figure 2). The saphenous branch of the YITA-SV was found patent in all patients except 1 . No failures were reported in the proximal tract of the ITA. Overall, the short-term YITA-SV graft patency was 22/25 (88\%).

All cases of ITA malfunction were reported in the part of the ITA distal to the SV anastomosis and always in patients in whom the artery was used to revascularize a coronary artery with $<70 \%$ stenosis $(6 / 10$ vs $0 / 15 ; P=.02)$.

Distal (but not proximal) ITA graft diameter was significantly influenced by target vessel stenosis (Figure 3). Mean proximal ITA diameter was $2.71 \pm 0.21 \mathrm{~mm}$ for patients with $<70 \%$ LAD stenosis versus $2.67 \pm 0.18 \mathrm{~mm}$ in patients with $>70 \%$ lesion $(P=.47)$; in contrast, distal ITA diameter was $1.78 \pm 0.18 \mathrm{~mm}$ for patients with $<70 \% \mathrm{LAD}$ stenosis versus $2.25 \pm 0.09 \mathrm{~mm}$ in patients with $>70 \%$ lesion $(P<.001)$.

\section{Clinical and Scintigraphic Status}

At the time of the study no patient had angina recurrence; stress myocardial scintigraphy demonstrated some degree of inducible ischemia in 11 patients and absence of ischemia in the remaining 14. All 7 patients with YITA-SV malfunction (6 ITAs and 1 great SV) had scintigraphic evidence of ischemia in the graft territory. In the remaining patients ischemia was localized in non- 


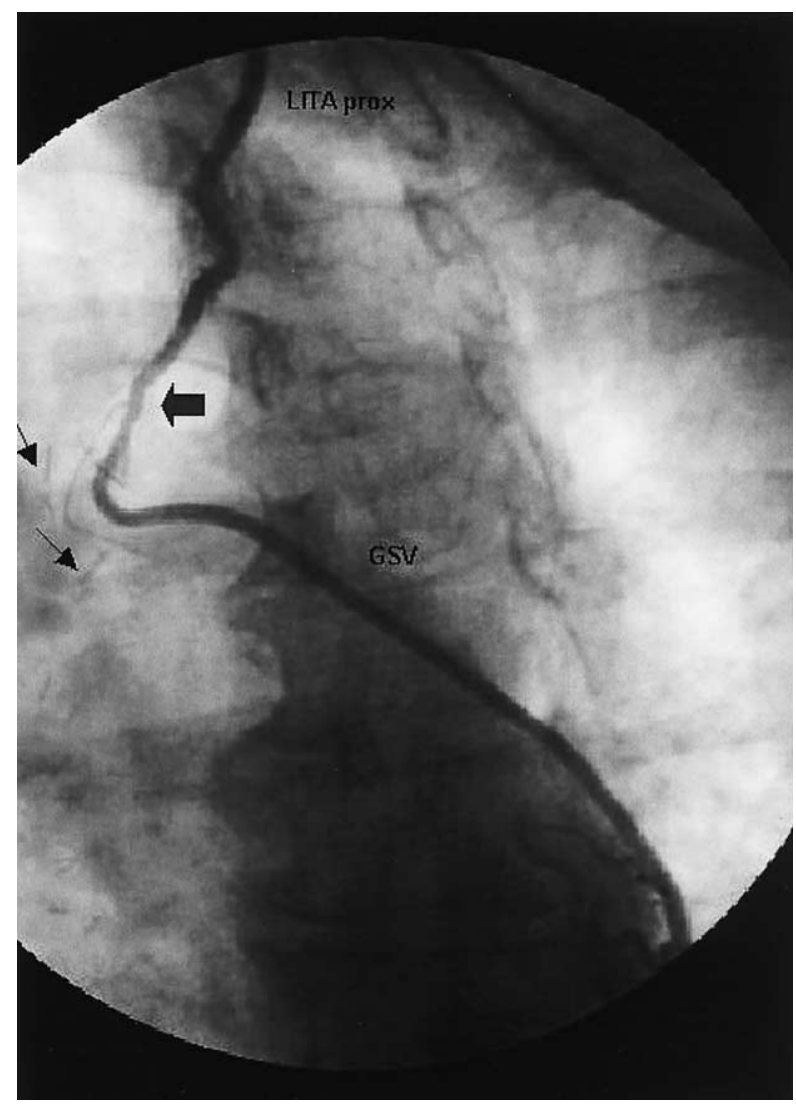

Figure 2. Occluded distal internal thoracic artery in a $Y$ internal thoracic artery-saphenous vein graft. The distal tract of the internal thoracic artery results occluded; of note, injection in the left coronary system consents visualization of the distal part of the internal thoracic artery indicating a patent distal anastomosis. Large arrow indicates the presumed level of thoracic artery-saphenous vein anastomosis, whereas small arrows indicate clips on the occluded portion of the internal thoracic artery. GSV, Great saphenous vein; LITA prox, proximal left internal thoracic artery.

grafted territories and related to the incompleteness of surgical revascularization. Due to poor functional capacity, old age, and absence of symptoms, no patient was submitted to further invasive procedure.

\section{Vasoreactivity}

YITA-SV reactions to endovascular vasoactive challenges are summarized in Table 3. Proximal and distal ITAs did not significantly react to serotonin infusion and showed significant dilatation after the administration of isosorbide dinitrate. The proximal part of the ITA also showed significant capacity of endothelium-dependent vasodilatation, whereas the distal tract of the artery dilated only slightly following acetylcholine challenge. The SV branch reacted to both serotonin and isosorbide dinitrate but did not show significant endothelium-mediated relaxation.

\section{Discussion}

Anastomosing the proximal portion of an SV to an ITA creates a "hybrid" arteriovenous graft that shares the biologic and functional characteristics of the best and the worst conduit used for CABG procedures. In the past it has been suggested that YITA-SV can represent the appropriate solution in cases of severe ascending aorta atherosclerosis, particularly when there is some contraindication to the use on non-left ITA arterial grafts. ${ }^{1-4}$

Our series reports the angiographic results and vasoreactive profile of 25 YITA-SVs at a mean interval of 2.5 years from surgery. We found that the overall patency $(22 / 25=88 \%)$ was lower than that reported for single ITA and for composite all-arterial conduits. ${ }^{6,7}$ The great majority of graft malfunctions (string or occlusion) were localized in the portion of the ITA distal to the SV anastomosis and were reported when the artery was used to revascularize target vessels with a moderate $(<70 \%)$ stenosis.

Theoretically YITA-SV represent an interesting model of ITA flow diversion. The SV graft can be regarded as a major ITA collateral branch whose caliber is significantly larger than that of the main artery itself. This situation creates the possibility of a significant flow diversion from the ITA to the SV; flow steal is even enhanced when the ITA is anastomosed to a coronary vessel with moderate stenosis. ITA side branch flow steal has been denied in single ITA grafts ${ }^{8,9}$; however, the diameter and length of the SV grafts proximally anastomosed to the artery are larger than those of the ITA side branches, and the YITA-SV represents a hemodynamic model that cannot be compared with the single ITA with its natural side branches.

Although in more physiologic situations the ITA is able to adapt to a wide flow range without occlusion, the hemodynamic condition created by the anastomosis of a large conduit to the proximal part of the artery renders YITA-SV more susceptible to the detrimental effect of chronic native competitive flow. It is interesting to note that this dependence of $\mathrm{Y}$ graft patency from flow competition has not been reported in angiographic series where the graft was constructed by anastomosing 2 arterial conduits (usually 2 ITAs or the ITA and a radial artery). ${ }^{6,7}$ It must be considered that the SV is larger and less reactive than all arterial conduits and is likely to offer less resistance to flow. It is then conceivable that flow diversion from the main stem of the Y graft is higher when the side branch is represented by an SV instead of a right ITA or a radial artery, rendering YITA-SV more vulnerable to native competitive flow.

The portion of the ITA distal to the SV also exhibited a lower capacity of endothelium-dependent vasodilatation (see Table 3); as it is well known that flow and shear stress are major determinant of nitric oxide (NO) production and endothelium-mediated dilatation, ${ }^{10}$ it seems highly likely 


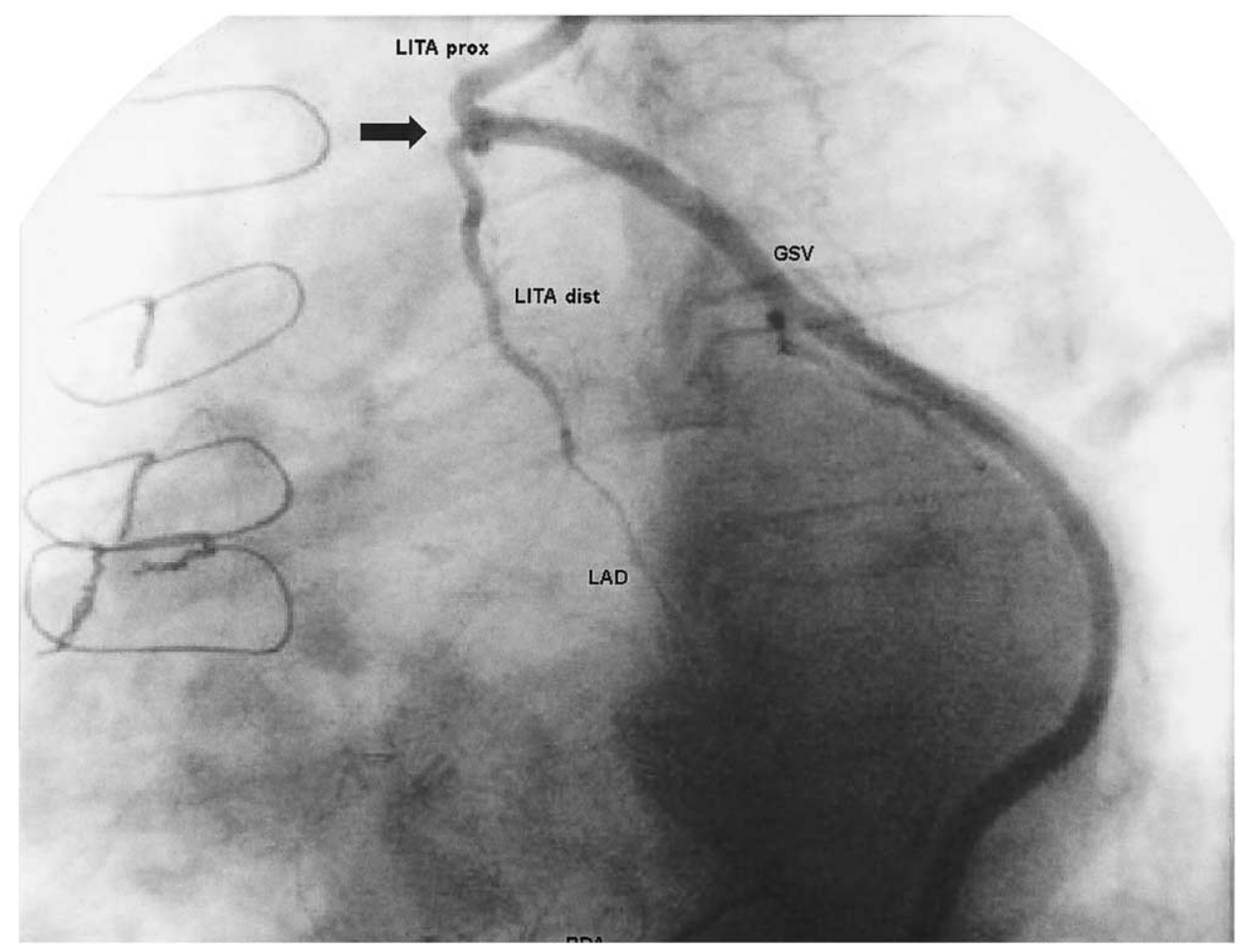

Figure 3. Caliber reduction between proximal and distal tract of the internal thoracic artery in an $Y$ internal thoracic artery-saphenous vein graft. Marked reduction of internal thoracic artery diameter is evident between the portion of the artery proximal and distal to the saphenous vein anastomosis. Arrow indicates thoracic arterysaphenous vein anastomosis. GSV, Great saphenous vein; LAD, left anterior descending artery; LITA prox, proximal left internal thoracic artery; LITA dist, distal left internal thoracic artery.

TABLE 3. Mean diameters after endovascular vasoactive challenges

\begin{tabular}{lllll}
\hline & Baseline & Acetylcholine & Serotonin & Isosorbide dinitrate \\
\hline Proximal ITA (mm) & $2.88 \pm 0.10$ & $2.96 \pm 0.11^{*}$ & $2.86 \pm 0.09$ & $2.98 \pm 0.11^{*}$ \\
Distal ITA (mm) & $2.11 \pm 0.05 \dagger$ & $2.13 \pm 0.03$ & $2.09 \pm 0.03$ & $2.23 \pm 0.06^{*}$ \\
Saphenous vein (mm) & $3.78 \pm 0.31 \ddagger$ & $3.74 \pm 0.33$ & $3.51 \pm 0.25^{*}$ & $4.01 \pm 0.20^{*}$ \\
\hline
\end{tabular}

ITA, Internal thoracic artery.

${ }^{*} P<.05$ vs baseline.

† $P<.001$ vs proximal ITA diameter.

$\ddagger P<.001$ vs proximal and distal ITA diameter.

that reduced NO synthesis due to low flow in the distal ITA could have played a causal role in graft malfunction and occlusion.

YITA-SV have been proposed for complex technical settings and in high-risk patients and, in this view, the suboptimal patency rate that we have found could be considered acceptable. However, our findings show that this type of graft configuration jeopardizes the distal tract of the ITA, placing at risk the most important myocardial territory. When adopting this type of conduit surgeons must be aware of this possibility and consider eventual alternative technical solutions.
In our institution after evaluation of the present data we have abandoned the use of these composite grafts. In the situations where we used YITA-SV we now adopt 2 different solutions:

- performance of an all-arterial Y graft using alternative arterial conduits such as the inferior epigastric or gastroepiploic arteries or even the radial or right ITAs if the risk associated with their use is judged less important than the possibility of performing 1 additional graft;

- incomplete revascularization by a single ITA graft on the LAD with eventual successive percutaneous treatment of the non-LAD vessels. ${ }^{11}$ 


\section{References}

1. Mills NL, Everson CT. Atherosclerosis of the ascending aorta and coronary artery bypass. Pathology, clinical correlates, and operative management. J Thorac Cardiovasc Surg. 1991;102:546-53.

2. Dietl CA, Madigan NP, Laubach CA, et al. Myocardial revascularization using the "no-touch" technique, with mild systemic hypothermia, in patients with a calcified ascending aorta. J Cardiovasc Surg. 1995; 36:39-44.

3. Murphy DA, Hatcher CR Jr. Coronary revascularization in the presence of ascending aortic calcification: use of an internal mammary artery-saphenous vein composite graft. J Thorac Cardiovasc Surg. 1984;87:789-91.

4. Brodman R, Robinson G. Internal mammary artery-saphenous vein composite conduit: an alternative for the proximal coronary anastomosis. Ann Thorac Surg. 1981;31:370-2.

5. Gaudino M, Trani C, Glieca F, et al. Early vasoreactive profile of skeletonized versus pedicled internal thoracic artery grafts. J Thorac Cardiovasc Surg. 2003;125:638-41.
6. Calafiore AM, Di Giammarco G, Luciani N, et al. Composite arterial conduits for a wider arterial myocardial revascularization. Ann Thorac Surg. 1994;58:185-90.

7. Weinschelbaum EE, Gabe ED, Macchia A, Smimmo R, Suarez LD. Total myocardial revascularization with arterial conduits: radial artery combined with internal thoracic arteries. J Thorac Cardiovasc Surg. 1997; 114:911-6.

8. Gaudino M, Serricchio M, Glieca F, et al. Steal phenomenon from mammary side branches: when does it occur? Ann Thorac Surg. 1998;66:2056-62.

9. Cohn WE, Ruel M, Zhang JP, Sellke FW, Johnson RG. Internal thoracic artery flow competition: studies in a canine H-graft model. Eur J Cardiothorac Surg. 2003;23:56-9.

10. Cooke JP, Rossitich E, Andon NA, Loscalzo J, Dzau VJ. Flow activates an endothelial potassium channel to release an endogenous nitrovasodilator. J Clin Invest. 1991;88:1663-70.

11. Gaudino M, Cellini C, Bruno P, Zimarino M, Possati G. Integrated approach in cases of multivessel disease and porcelain aorta. J Card Surg. 1998;13:140-2. 